\title{
Historiographie des sciences et des techniques au Canada
}

\section{Raymond Duchesne}

Volume 35, numéro 2, septembre 1981

URI : https://id.erudit.org/iderudit/303950ar

DOI : https://doi.org/10.7202/303950ar

Aller au sommaire du numéro

Éditeur(s)

Institut d'histoire de l'Amérique française

ISSN

0035-2357 (imprimé)

1492-1383 (numérique)

Découvrir la revue

Citer cet article

Duchesne, R. (1981). Historiographie des sciences et des techniques au Canada. Revue d'histoire de l'Amérique française, 35(2), 193-215.

https://doi.org/10.7202/303950ar d'utilisation que vous pouvez consulter en ligne.

https://apropos.erudit.org/fr/usagers/politique-dutilisation/ 


\title{
HISTORIOGRAPHIE DES SCIENCES ET DES TECHNIQUES AU CANADA
}

\author{
RAYMOND DUCHESNE \\ Institut d'histoire et de \\ sociopolitique des sciences \\ Université de Montréal
}

En novembre 1978, la Conférence de Kingston sur l'étude de l'histoire de la science et de la technologie canadiennes réunissait près de 150 personnes (historiens, sociologues, bibliothécaires, archivistes, éditeurs et simples amateurs), afin de discuter de l'état des recherches et de l'enseignement dans ce domaine et des mesures à prendre pour préserver et faire mieux connaître l'héritage scientifique et technologique du Canada. Les actes de la Conférence, publiés récemment (Richard A. Jarrell et Norman R. Ball, ed., Science, Technology, and Canadian History, Waterloo, 1980), rendent fidèlement compte des discussions qui ont eu lieu au sujet de la nature et de l'état de conservation des archives canadiennes dans ce domaine, de la valeur des collections déposées dans les musées, du rôle des organismes gouvernementaux dans le financement et l'encadrement de la recherche historique, de la disponibilité de sources et d'instruments bibliographiques pouvant servir à l'enseignement de l'histoire des sciences et des techniques au Canada, etc.

Plusieurs des idées et des propositions formulées à cette occasion continuent d'être débattues. D'autres ont donné lieu à des réalisations concrètes ou ont trouvé un commencement d'exécution. Le History of Science and Technology in Canada Bulletin ${ }^{1}$, un modeste «newsletter» qui circulait depuis 1976, s'est transformé au début de 1981 en une véritable revue savante dont les pages sont ouvertes aux études originales sur tout aspect de l'histoire des sciences, des techniques et de la médecine au Canada. Longtemps regroupés au sein de la Société canadienne d'histoire et de philosophie des sciences, les «canadianistes» viennent de s'en détacher afin de former l'Association pour l'histoire de la science et de la technologie au Canada, société à laquelle sont invités à se joindre les historiens amateurs comme les historiens professionnels. Enfin,

1 Désigné par la suite comme le HSTC Bulletin. 
une seconde Conférence sur l'histoire des sciences et des techniques au Canada doit avoir lieu à Kingston en novembre 1981, afin, sans doute, de mesurer le chemin parcouru depuis 1978 et de voir de quel côté il faudrait maintenant faire porter les efforts.

$\mathrm{Au}$ milieu de ces diverses entreprises et peut-être en raison même de l'agitation qui accompagne généralement l'émergence d'une nouvelle discipline, on a quelque peu négligé de réfléchir sur ce que nous appellerons ici les fondements problématiques de l'histoire des sciences et des techniques au Canada, de même que l'on a négligé de montrer comment celle-ci pouvait se rapporter aux grandes questions qui préoccupent les chercheurs des domaines plus classiques de l'histoire canadienne, qu'il s'agisse de l'histoire politique, de l'histoire économique et sociale, ou encore, de l'histoire de la culture. Pour cette raison, l'histoire des sciences et des techniques continue d'être un domaine sans grande unité au plan des interrogations, des méthodes ou des concepts, et un genre marginal dans l'historiographie canadienne, en dépit de l'intérêt considérable qu'elle suscite actuellement. Dans cet essai, nous nous proposons de passer en revue les travaux anciens et récents qui forment le corps principal de l'historiographie des sciences et des techniques au Canada. Le but premier de cet inventaire est de faire connaître, surtout à ceux qui n'ont aucune familiarité avec le domaine, les sources biographiques et bibliographiques, les ouvrages de synthèse et l'état d'avancement des recherches. En même temps, nous voudrions faire un bilan critique des travaux plus anciens, qui constituent en quelque sorte l'héritage légué par les travailleurs de la première heure à la discipline en train de naître, et identifier les tendances dominantes de la recherche actuelle.

Peut-être verra-t-on mieux alors à quoi peuvent servir les instruments - conférences, revue savante et société - dont les historiens de la science et de la technologie au Canada ont choisi de se doter. Peut-être verra-t-on mieux aussi en quoi les problèmes de l'histoire des sciences et des techniques sont intimement liés à ceux de l'histoire économique, sociale et culturelle du Canada.

\section{L'histoire des sciences et des techniques au Canada: une longue tradition historiographique}

Que l'histoire des sciences et des techniques soit encore un genre marginal de l'histoire du Canada, on peut difficilement le nier devant la place qui lui est réservée dans les principales revues historiques et dans les grands répertoires bibliographiques. Malgré quelques exceptions notables (qui seront pour la plupart citées dans la suite du texte), relativement peu d'études d'histoire des sciences et des techniques ont été publiées dans la Revue d'histoire 
de l'Amérique française, Histoire sociale ou la Canadian Historical Review. Ni dans la bibliographie publiée régulièrement par la $R H A F$, ni dans celle de la $C H R$ a-t-on jugé à propos de créer une rubrique consacrée aux travaux d'histoire des sciences et des techniques. Les ouvrages de référence des historiens canadiens (A. Beaulieu, J. Hamelin, B. Bernier, Guide d'histoire du Canada, Québec, 1969; R. Durocher et P.-A. Linteau, Histoire du Québec. Bibliographie sélective, 1867-1970, Trois-Rivières, 1976; R. Boily, Québec 1940-1969: bibliographie, Montréal, 1971; C. Thibault, Bibliographia Canadiana, Toronto, 1973; etc.) se contentent habituellement de citer les titres les plus anciens et les mieux connus: le volume du cinquantenaire de la Société royale du Canada (Fifty Years Retrospect, 1882-1932, Ottawa, 1932), les collections d'études historiques rassemblées par le Dr. H.M. Tory ( $A$ History of Science in Canada, Toronto, 1939) et par G.F.G. Stanley (Pioneers of Canadian Science, Toronto, 1939), et l'esquisse de Cyrias Ouellet (La vie des sciences au Canada français, Québec, 1964).

Dans les universités canadiennes, l'enseignement de l'histoire des sciences et des techniques au Canada n'a guère progressé au cours des dernières années, en dépit des souhaits exprimés par les participants à la Conférence de Kingston et des efforts déployés en ce sens depuis ${ }^{2}$. Pourtant, il existe à l'intention de ceux qui pourraient vouloir créer un cours de premier cycle ou un séminaire avancé dans ce domaine quelques outils bibliographiques et historiques de base, notamment une anthologie consacrée à l'histoire des sciences au Canada et une autre consacrée à l'histoire des techniques (T.H. Levere et R.A. Jarrell, ed., A Curious Field-Book. Science and Society in Canadian History, Toronto, 1974; B. Sinclair, N.R. Ball et J.O. Petersen, ed., Let Us Be Honest and Modest: Technology and Society in Canadian History, Toronto, 1974). En fait, le peu de place qu'occupe l'histoire des sciences et des techniques dans l'historiographie canadienne paraît d'autant plus surprenant que l'ensemble des travaux qui ont été consacrés au passé scientifique et technique des Canadiens représente une véritable tradition de recherches historiques.

La richesse de cette tradition a été partiellement mise en lumière par certains répertoires bibliographiques spécialisés publiés au cours des deux dernières décennies. Le plus ancien de

2 En 1978, seuls l'Institute for the History and Philosophy of Science and Techno$\log y$ de l'Université de Toronto et l'Institut d'histoire et de socio-politique des sciences de l'Université de Montréal offraient des cours avancés sur le sujet. La situation n'a guère changé, mais on remarque ici et là quelques initiatives. Â l'Université d'Ottawa, par exemple, on a établi un cours intitulé "Technology and Society in North American History». Ailleurs, des éléments de l'histoire canadienne sont désormais intégrés à des cours d'histoire des sciences, des techniques et de la médecine. 
ces répertoires, mais qui n'a rien perdu de son utilité pour l'historien des sciences et des techniques, comme d'ailleurs pour le spécialiste des questions d'éducation auquel il était destiné, est celui de R.S. Harris et de A. Tremblay (A Bibliography of Higher Education in Canada, Toronto, 1960, ouvrage complété par des Supplements, parus en 1965 et en 1970, et poursuivi annuellement par la bibliographie de STOA: The Canadian Journal of Higher Education). En 1968, la Bibliothèque scientifique nationale, à Ottawa, publiait la première édition d'une bibliographie consacrée à $L a$ politique des sciences, la recherche et le développement au Canada, répertoriant l'ensemble des publications (comptes rendus, volumes, articles, documents officiels, etc.) parues depuis 1935, y compris celles à caractère historique. Une édition révisée a été publiée en 1970. Par la suite, la Bibliothèque, devenue entretemps partie de l'Institut canadien de l'information scientifique et technique, a tenu compte de l'évolution de la littérature en faisant paraître divers suppléments (Supp. I (1970-1972), Supp. II (19721975), Supp. III (1975-1977), Supp. IV (1977-1979).

Malgré le soin avec lequel elles ont été préparées et le vaste champ qu'elles couvrent, les deux bibliographies précédentes n'étaient pas taillées sur mesure pour les historiens des sciences et des techniques: aussi a-t-on vu paraître depuis une bibliographie spécialement consacrée à l'histoire scientifique et technique du Canada ( $A$ Bibliography for Courses in the History of Canadian Science, Medicine, and Technology, Comp. par R.A. Jarrell et Arnold Roos, Thornhill, 1979). La première édition de l'ouvrage, dont cet essai fait ample usage, inventorie plus de 1000 titres et, sans être exhaustive, permet d'estimer en même temps la diversité des travaux anciens et les orientations de la recherche actuelle. Destinée à l'enseignement universitaire, mais susceptible de servir à quiconque voudrait entreprendre des recherches sur un aspect particulier de l'histoire des sciences, des techniques ou de la médecine au Canada, cet instrument bibliographique, dans l'intention de ses auteurs, est appelé à connaître de nombreuses révisions de manière à suivre la croissance et le perfectionnement de l'historiographie.

Enfin, Bertrum H. Macdonald et R. Alan Richardson, de l'University of Western Ontario, ont compilé un Preliminary Bibliographical Inventory of Sources in the History of Science, Technology and Medicine in Canada to the Twentieth Century. La première version de cet ouvrage considérable, comprenant près de 3000 références bibliographiques classées selon les auteurs et selon les sujets, circule présentement parmi un groupe restreint d'historiens des sciences et des techniques. Avec les corrections et les 
additions qu'elle est appelée à recevoir, cette somme bibliographique de la contribution du Canada à la connaissance scientifique, technologique et médicale universelle deviendra sans aucun doute un instrument essentiel de la recherche historique.

Traditionnellement, ceux qui se sont intéressés à l'histoire des sciences et des techniques au Canada se sont surtout efforcés de documenter la biographie des savants et des inventeurs du passé. Aussi ne doit-on pas se surprendre de trouver dans les grands répertoires biographiques canadiens de nombreuses esquisses de la vie et de l'oeuvre des principaux personnages de notre histoire scientifique et technique. Dans les quatre premiers volumes du Dictionnaire biographique du Canada, qui, rappelons-le, couvrent la période s'étendant des premières reconnaissances des côtes de l'Amérique du Nord par les Islandais jusqu'à 1800, figurent en bonne place les explorateurs et les cartographes, comme William Baffin, Sir Martin Frobisher, Louis Jolliet, les hydrographes et les ingénieurs militaires du Régime français, comme Jean-BaptisteLouis Franquelin et Gaspard-Joseph Chaussegros de Léry, les voyageurs et les chroniqueurs qui ont laissé des descriptions de l'histoire naturelle de l'Amérique du Nord, comme Nicolas Denys, Pierre Boucher, le père Pierre-François-Xavier de Charlevoix, le disciple de Linné, Pehr Kalm, qui visita la Nouvelle France en 1747 , le père Joseph-François Lafitau, missionnaire qui fit la découverte du ginseng canadien, les naturalistes et les herborisateurs, comme Michel Sarrazin, Jean-François Gaulthier, l'abbé Jean-Baptiste Gosselin et d'autres moins connus, qui fournirent en spécimens du Nouveau Monde les savants d'Europe, en particulier ceux de l'Académie des Sciences et du Jardin du Roi à Paris. Certaines de ces biographies, celles, notamment, de Sarrazin par Jacques Rousseau et de Gaulthier par Bernard Boivin, dépassent largement le personnage et constituent de véritables descriptions du milieu social et scientifique.

Dans les deux volumes du Dictionnaire qui se rapportent au $\mathrm{XIX}^{\mathrm{e}}$ siècle, on retiendra, parmi d'autres, les biographies de géologues, tels Abraham Gesner, Frederick Henry Baddeley et William Logan, d'explorateurs, tels Sir John Richardson et Sir Edward Belcher, la biographie du premier paléontologiste de la Commission géologique, Elkanah Billings, celles de quelques professeurs de sciences, tels James Bovell, du Trinity College de Toronto, de William Hincks, premier professeur d'histoire naturelle au University College de la même ville, et de l'abbé Louis-Ovide Brunet, professeur de botanique à l'Université Laval. On retiendra également la notice consacrée à James Hodge, l'ingénieur en chef du pont Victoria, et celles consacrées à des naturalistes amateurs dis- 
tingués tels le Dr Jean-Baptiste Meilleur et William Sheppard. Enfin, les éditeurs du Dictionnaire ont annoncé la publication, dans les volumes à venir, des biographies de personnages de premier plan de l'histoire canadienne des sciences et des techniques, par exemple, Edward Sabine, George Lawson, l'abbé Léon Provancher et Thomas Sterry Hunt.

Le Dictionnaire biographique du Canada constitue donc une source importante pour la biographie des savants et des ingénieurs qui ont oeuvré en sol canadien depuis les débuts de la colonisation européenne. Cependant, le chercheur se verra souvent contraint de pousser ses recherches au-delà des volumes du $D B C$ et de faire appel à des répertoires biographiques spécialisés. Le Dictionary of Scientific Biography, dont les derniers volumes et l'index viennent tout juste de paraître, comprend la biographie de plusieurs grandes figures de l'histoire canadienne et se révèle particulièrement utile pour les périodes que ne couvre pas encore le $D B C$. Par exemple, on peut y trouver d'excellents articles consacrés à John William Dawson, paléobotaniste distingué et Principal de l'Université McGill pendant presque toute la seconde moitié du XIX ${ }^{\mathrm{e}}$ siècle, à Frederick G. Banting, co-découvreur avec Charles Best de l'insuline, à l'astronome John Stanley Plaskett et au chimiste William L. Miller. Parfois, les chercheurs trouveront dans les histoires de certaines disciplines scientifiques en Amérique du Nord une abondance de matériaux biographiques concernant des savants canadiens, ceux-ci se trouvant assez souvent confondus avec les hommes et les femmes de science américains. C'est le cas, notamment, des entomologistes (E.O. Essig, A History of Entomology, New York, 1931; Arnold Mallis, American Entomologists, New Jersey, 1971) et des physiciens (M. Bunge et W.R. Shea (ed.) Rutherford and Physics at the Turn of the Century, Londres, 1979).

Quelques revues, comme les Mémoires de la Société royale du Canada, le Naturaliste canadien ou le Canadian Entomologist, fondées toutes deux en 1868, contiennent de nombreuses esquisses biographiques et notices nécrologiques consacrées aux membres éminents de la communauté scientifique canadienne. Fort inégales, ces notices constituent parfois un utile point de départ, surtout lorsqu'elles comprennent une bibliographie de l'oeuvre scientifique du personnage ou un jugement critique sur cette oeuvre.

Outre les articles des dictionnaires biographiques et les notices, il existe, bien sûr, de nombreux travaux plus élaborés sur la vie et l'oeuvre de certains savants canadiens. Parmi les essais autobiographiques méritant d'être signalés, on compte celui de John Macoun, un des plus importants botanistes canadiens du siècle dernier (Autobiography, Ottawa, 1922), celui du Dr Wilder Pen- 
field, neurochirurgien et neuropathologiste éminent (No Man Alone: A Neurosurgeon's Life, Boston, 1977), celui du physicien Leopold Infield (Why I Left Canada: Reflection on Science and Politics, édité par L. Pyenson, Montréal, 1978) et l'autobiographie de J.W. Dawson (Fifty Years of Work in Canada, Londres, 1901). Ce dernier a eu également son biographe (C.E. O'Brien, Sir William Dawson. A Life in Science and Religion, Philadelphie, 1971), ce qui doit être considéré comme une indication de l'importance du rôle qu'il a joué dans l'institutionnalisation des sciences au Canada anglais au XIX ${ }^{\mathrm{e}}$ siècle. Son fils, George Mercer Dawson, qui fit une carrière remarquable à la Commission géologique du Canada, a aussi retenu l'attention des historiens (L. WinslowSpragge, Life and Letters of George Mercer Dawson, Montréal, 1962; et J.C. Barkhouse, George Dawson, the Little Giant, Toronto, 1974). Le genre biographique a été illustré, en outre, par les travaux de B.J. Harrington sur Sir William Logan, premier directeur de la Commission géologique, (Life of Sir William Logan, Montréal, 1883), du Chanoine V.-A. Huard sur l'abbé Léon Provancher (La vie et l'oeuvre de l'abbé Léon Provancher, Québec, 1926), du chanoine Lionel Groulx et de Roland Lamontagne sur La Galissonière, gouverneur de la Nouvelle-France de 1747 à 1749 et protecteur des naturalistes comme Gaulthier et Kalm (respectivement, Roland-Michel Barrin de La Galissonière, Québec, 1970 et La Galissonière et le Canada, Paris, 1962), etc.

Enfin, des travaux plus récents, dont la qualité et l'intérêt doivent être soulignés, indiquent que la recherche biographique est toujours populaire auprès des historiens et qu'elle peut contribuer grandement à éclairer l'histoire des sciences et des techniques au Canada. Parmi ces travaux plus récents, on remarque les biographies de Sir John Richardson, le compagnon de Sir John Franklin dans ses explorations du Nord-Ouest (R.E. Johnson, Sir John Richardson. Arctic Explorer, Natural Historian, Naval Surgeon, Londres, 1976), de l'ingénieur Sandford Fleming, dont la carrière fut étroitement liée au développement ferroviaire du Canada (L. Green, Sandford Fleming, Toronto, 1980) et de Henry Youle Hind, professeur d'histoire naturelle au Trinity College de Toronto (W.L. Morton, Henry Youle Hind, Toronto, 1980).

Après la vie et l'oeuvre des savants et des inventeurs, c'est sans doute les principales institutions où se faisaient l'enseignement et la recherche scientifiques qui ont le plus fréquemment retenu l'attention des historiens canadiens. Par exemple, la question de l'enseignement des sciences dans les collèges classiques du Canada français, d'abord discutée dans les travaux originaux de Léon Lortie («Les sciences à Montréal et à Québec au XIX ${ }^{\mathrm{e}}$ siè- 
cle», Action universitaire 1(3)(1936): 46 et «Les mathématiques de nos ancêtres», Mémoires de la Société royale du Canada (MSRC) I, 49(1955): 31-45), a été reprise dans des analyses plus vastes; en particulier, dans celles de Claude Galarneau («L'enseignement des sciences au Québec et Jérôme Demers», dans Mélanges d'histoire du Canada français, Ottawa, 1978: 84-94 et surtout dans Les collèges classiques au Canada français, Montréal, 1978). Les Mechanics' Institutes, qui semblent avoir proliféré au Canada anglais au cours du siècle dernier, continuent également d'être l'objet de recherches historiques (C.B. Ferguson, "Mechanics' Institutes in Nova Scotia», Bull. Public Archives of Nova Scotia 14 (1960); «Mechanics' Institutes in British Columbia», Continuous Learning 3 (10)(1971): 126-130; J.A. Eadie, "The Napanee Mechanics' Institute», Ontario History 4 (68)(1976): 209-221; etc.).

Cependant, le développement des recherches scientifiques a surtout eu pour cadre les institutions d'enseignement supérieur; par conséquent, c'est dans les travaux historiques consacrés à ces institutions, universités et écoles de génie, que l'historien des sciences trouvera la matière la plus abondante. S'ajoutant aux ouvrages généraux de Robin Harris ( $A$ History of Higher Education in Canada, 1663-1960, Toronto, 1976) et de L.-P. Audet (Histoire de l'enseignement dans la province de Québec, Montréal, 1971), il existe des études consacrées à la plupart des grandes universités canadiennes et même à certains départements; citons, à titre d'exemples, celles de E.H. Craigie ( $A$ History of the Department of Zoology of the University of Toronto, Toronto, 1966), de J.F. Snell et al. (Macdonald College of McGill University: A History for 1904-1955, Montréal, 1963), de C.R. Young (Early Engineering Education at Toronto, 1851-1919, Toronto, 1958) et de Stanley B. Frost (McGill University: For the Advancement of Learning, 1801-1895, Montréal, 1980). Pour diverses raisons, ces études présentent un intérêt tout particulier pour les historiens des sciences et des techniques. Malheureusement, il subsiste dans l'histoire des institutions d'enseignement scientifique du Canada des «trous» importants: par exemple, l'histoire de l'Ecole polytechnique de Montréal est encore à écrire. Dans d'autres cas, les historiens ont largement ignoré les sciences pures et appliquées dans leur compte rendu du développement des institutions (cf. H. Neatby, Queen's University: 1841-1917, Montréal, 1979). En ce qui concerne les universités des provinces de l'Ouest et de la Colombie britannique, beaucoup plus récentes que celles de l'Est, la majeure partie du travail historique reste encore à accomplir.

On peut faire une observation semblable dans le cas des sociétés savantes du Canada: pour la plupart d'entre elles, il n'existe 
encore que ces études partielles ou essentiellement descriptives que les historiens de la première génération nous ont léguées. Ainsi, sur l'histoire de la Literary and Historical Society of Quebec, une des plus anciennes sociétés savantes du Canada et qui, en dépit de son nom, a fait une large place aux sciences naturelles, on ne trouvera que le volume publié lors du centenaire de l'institution (L.H.S.Q., Centenary Volume, 1824-1924, Québec, 1924) et des travaux universitaires, plus récents peut-être, mais peu accessibles (Laura I. Bancroft, The Literary and Historical Society of Quebec, Mémoire de M.A., Université Laval, 1950 et Ginette Bernatchez, La Société littéraire et historique de Québec, Mémoire de M.A. (histoire), Université Laval, 1980). Il en va de même pour la Société royale du Canada, l'ouvrage de base restant le volume du cinquantenaire (Fifty Years Retrospect, 1882-1932, op. cit.), pour le Royal Canadian Institute de Toronto (W.S. Wallace, ed.), Royal Canadian Institute Centenary Volume, 1849-1949, Toronto, 1949) et pour l'Entomological Society of Canada (G.B. Wiggins ed.), Centennial of Entomology in Canada, 1863-1963, Toronto, 1966). Pour beaucoup de sociétés, enfin, comme l'ACFAS, la Natural History Society of Montreal ou le Canadian Engineering Institute, nous ne disposons même pas de telles esquisses historiques qui permettraient d'identifier les principaux acteurs de leur histoire ou de marquer les grandes étapes de leur développement. Il semble y avoir là un domaine tout désigné pour de nouvelles recherches historiques, à plus forte raison si l'on songe, d'une part, au rôle joué par certaines d'entre elles dans l'histoire des sciences au Canada et, d'autre part, au fait que plusieurs ont soigneusement conservé leurs archives depuis leur origine.

Lorsque, des sociétés savantes, on se tourne vers les agences scientifiques et techniques de l'Etat fédéral, la rareté des études historiques fait aussitôt place à l'abondance. En effet, la plupart des grandes agences ont déjà été l'objet de travaux, à commencer par la Commission géologique (Morris Zaslow, Reading the Rocks. A Story of the Geological Survey of Canada, 1842-1972, Ottawa, 1975), le Fisheries Research Board (Kenneth Johnstone, The Aquatic Explorers. A History of the Fisheries Research Board of Canada, Toronto, 1977), les différents services de topographie, de cartographie et d'arpentage du Dominion (Don W. Thompson, L'homme et les méridiens, Ottawa, Vol. I (1966), Vol. II (1973) et les organismes chargés, depuis le XIX ${ }^{\mathrm{e}}$ siècle, d'établir l'heure locale dans les différentes parties du pays (M.M. Thompson, The Beginning of the Long Dash. A History of Timekeeping in Canada, Toronto, 1978). Cependant, aucune agence gouvernementale n'a vu son histoire fouillée avec tant d'acharnement, ni par autant de chercheurs, que le National Research Council of 
Canada, institution fondée en 1916. Parmi les études les plus anciennes consacrées au $N R C$, on peut citer celles de W. Eggleston (Scientists at War, Toronto, 1950) et de Mel Thistle (The Inner Ring: The Early History of the National Research Council of Canada, Toronto, 1966). Comme nous le verrons plus loin, ces premiers travaux ont ouvert la voie à des études plus récentes. L'attention accordée par les historiens au NRC comme aux autres agences gouvernementales témoigne essentiellement de leur importance dans le développement des recherches scientifiques au Canada et dans l'institutionnalisation des rôles du scientifique et de l'ingénieur. Aussi peut-on s'étonner que l'histoire de certaines agences, dont l'importance ne le cède en rien à celle d'institutions abondamment étudiées, ait été largement négligée jusqu'ici: par exemple, l'histoire de l'Atomic Energy of Canada Ltd., société de la Couronne qui est responsable depuis 1952 du développement de l'énergie nucléaire à des fins pacifiques et qui s'est illustrée notamment par la mise au point du réacteur CANDU, n'a pas encore été écrite, pas plus d'ailleurs que celle des Dominion Experimental Farms, créées à la fin du XIX ${ }^{\mathrm{e}}$ siècle et dont l'existence a grandement contribué à l'essor de l'agriculture canadienne. Bref, l'histoire institutionnelle des sciences au sein de l'appareil de l'État fédéral est considérablement avancée dans son ensemble, ce qui concourt à rendre plus évident encore le retard pris dans quelques cas.

Du côté de l'histoire des disciplines et des techniques particulières, l'historiographie traditionnelle a accumulé des travaux fort divers, dont nous ne présenterons ici qu'un échantillon. Signalons d'abord que, pour le meilleur ou pour le pire, les historiens américains n'ont généralement pas hésité à inclure les savants et les institutions du Canada dans leurs inventaires du développement des disciplines scientifiques en Amérique du Nord. Aussi peut-on trouver dans des "classiques» de l'histoire des sciences en Amérique, tels, par exemple, George Brown Goode ( $«$ The Beginnings of Natural History in America», Proc. Biological Society of Washington (1886): 35-105) ou A.D. Rodgers III (American Botany 1873-1892: Decades of Transition, Princeton, 1944), une ample moisson de renseignements relatifs à des naturalistes ayant fait des recherches sur le territoire actuel du Canada depuis le $\mathrm{XVI}^{\mathrm{e}}$ siècle. Des travaux plus récents, tels ceux de Cecil S. Schneer ( $«$ The Great Taconic Controversy», Isis 69(1978): 173-191) et de L. Badash (Radioactivity in America: Growth and Decay of a Science, Baltimore, 1979) indiquent que la frontière politique séparant les ÉtatsUnis du Canada n'a pas d'équivalent dans le champ des disciplines, pas plus pour les historiens actuels que pour leur devanciers. 
Bien sûr, l'«impérialisme» des historiens américains n'a pas empêché les Canadiens de s'intéresser à l'histoire des disciplines et de produire, dans certains cas, des travaux sinon "définitifs», du moins d'une incontestable utilité: citons des ouvrages de C.J.S. Warrington et de R.V.V. Nicholls ( $A$ History of Chemistry in Canada, Toronto, 1949), de R. Glen («Entomology in Canada up to 1956», Canadian Entomologist 88 (7)(1956): 290-371); de Vianney Legendre («Limnology in Quebec», dans D.G. Fry (ed.), Limnology in North America, Madison, 1963), de J.E. Kennedy ( Our Heritage in Canadian Astronomy», Journal of the Royal Astronomical Society 66 (1972): 83-98, d'E.G. Young (The Development of Biochemistry in Canada, Toronto, 1976), etc. Il importe de souligner qu'assez traditionnellement, l'histoire des disciplines a été l'oeuvre des praticiens eux-mêmes. De ce fait, ce que l'interprétation historique gagnait de la familiarité de l'auteur avec l'aspect conceptuel ou technique de son sujet, elle le perdait parfois au plan de l'analyse des rapports entre le développement scientifique proprement dit et le contexte social et culturel: en histoire des sciences, l'introspection a ses défauts comme ses qualités. Il n'a pas toujours été possible, non plus, aux «gens du métier» qui se penchaient sur l'histoire de leur discipline, d'éviter de tomber dans des travers tels que l'idéalisation des personnages historiques et des événements, ou l'adoption d'un point de vue "whiggish» sur le développement des institutions et des concepts.

Au passage, on peut ajouter que ce sont là des fautes qu'ont su mieux éviter les praticiens des sciences sociales et humaines qui ont entrepris de faire l'histoire de leur spécialité respective. Pour des raisons qui tiennent sans doute pour beaucoup au processus de constitution de ces disciplines et à leur statut épistémologique, les «historiens» des sciences sociales et humaines se sont montrés passablement critiques des travaux de leurs devanciers. On peut en juger en examinant, par exemple, les analyses de Carl Berger (The Writing of Canadian History. Aspects of English-Canadian Historical Writing, 1900-1970, Toronto, 1976) et de Serge Gagnon («Historiographie canadienne ou les fondements de la conscience nationale», dans A. Beaulieu et al., Guide d'histoire du Canada, op.cit., : 1-61) sur l'évolution de l'historiographie, ou encore celles des sociologues («La sociologie au Québec», numéro thématique de Recherches sociographiques 2-3 (15)(1974) et Sociologie et Sociétés 12 (2)(1980).

Enfin, derniers objets traditionnels de l'histoire des sciences au Canada, après les savants, les institutions et les disciplines, les grands événements - découvertes, inventions ou controverses ayant marqué le développement des sciences au Canada ont fixé 
l'attention des chercheurs. Certains événements n'ont eu qu'un intérêt local comme, par exemple, le débat entourant le transformisme en Ontario et au Québec au XIX siècle (P. Roome, "The Darwin Debate in Canada, 1860-1880», dans Science, Technology, and Culture in Historical Perspective, Calgary, 1976 :183-205). Cependant, l'historiographie a fait une plus large place aux recherches et aux découvertes qui dépassaient le contexte proprement canadien et qui ont eu une résonance ou une signification particulière dans l'histoire universelle des sciences et des techniques: citons, à cet égard, la controverse sur la nature, organique ou minéralogique, de l'Eozoön canadense, où se trouvèrent opposés des géologues et des paléontologistes du Canada, de l'Europe et des Etats-Unis (C.E. O’Brien, «Eozoön canadense: 'Dawn Animal of Canada'», Isis 61(1970):206-223), les expériences de Rutherford sur la désintégration des éléments radioactifs, expériences réalisées à l'Université McGill au début du XX $\mathrm{XX}^{\mathrm{e}}$ siècle (T.J. Trenn, The Self Splitting Atom: A History of the Rutherford-Soddy Collaboration, Londres, 1978; M. Bunge et W.R. Shea, Rutherford and Physics..., op. cit.) ou les travaux de Best et de Banting à l'Université de Toronto.

Citons également, dans le domaine de l'histoire des techniques, l'intérêt considérable suscité par l'invention du téléphone par Alexander Graham Bell. Comme on peut s'en douter, il existe sur chacun de ces grands épisodes de l'histoire des sciences et des techniques au Canada une telle quantité d'études qu'il est impossible d'entreprendre ici d'en faire la revue. Sur ce point, contentonsnous de remarquer que, par le passé, on a trop souvent été porté à réduire l'histoire des sciences au Canada à ces quelques événements d'une importance universelle, négligeant du même coup de dégager pleinement le sens du long processus de développement des pratiques scientifiques et techniques dans la société canadienne.

L'examen rapide des travaux produits jusqu'ici par les historiens des sciences et des techniques au Canada éclaire l'ampleur et la richesse de cette tradition historiographique: répertoires bibliographiques, biographies, études consacrées à des institutions d'enseignement et de recherche, analyses du développement des disciplines et des spécialités techniques, etc., tout cela couvre l'ensemble de l'histoire du Canada, depuis ses origines, ainsi que les principaux champs du savoir et de l'activité technique. Cependant, devant une telle diversité de sujets et de styles, on peut se demander s'il s'agit bien là d'une tradition! Qu'ont donc en commun les travaux cités jusqu'à présent, qui nous autorise à parler d'une histoire «traditionnelle» des sciences et des techniques au Canada? 
Sans prétendre appliquer à tous et à chacun des travaux cités précédemment cette généralisation, nous croyons pouvoir soutenir que la production historique passée peut se rapporter à une intention fondamentale, la défense et l'illustration de la «Science» au Canada. Ce qui fait une tradition de l'ensemble des études historiques consacrées au développement des sciences et des techniques au Canada, c'est l'uniformité et la permanence des objectifs que se sont donnés les différents auteurs, subordonnant l'analyse du passé aux exigences idéologiques présentes de la pratique des sciences et à l'affermissement du rôle du «scientifique» par rapport soit à l'État et au champ politique, soit à la société dans son ensemble. Bref, l'histoire des sciences et des techniques au Canada a eu traditionnellement pour fin de servir la «Science», ou, plus concrètement, l'image et les intérêts de certains groupes sociaux constitués autour des activités d'enseignement et de recherche ou étroitement associés à celles-ci. Quelques exemples peuvent contribuer à éclairer et à appuyer cette interprétation de l'oeuvre de nos devanciers.

On peut d'abord observer que de nombreuses recherches historiques ont été commandées par la pratique même des sciences. L'hagiographie est un genre qui a été abondamment cultivé, permettant d'offrir aux chercheurs du présent des exemples capables de les inspirer, entretenant le respect des pionniers et des précurseurs et alimentant, à partir du passé, toute cette mythologie du Savant et de la Science si nécessaire au bon fonctionnement des institutions scientifiques. Parfois, les historiens se sont donné des tâches plus difficiles, comme de trancher de délicates questions de priorité des découvertes, de rétablir dans la mémoire historique tel ou tel savant dont l'oeuvre avait été méconnue ou sous-estimée (voir, par exemple, J. Rousseau et W.G. Dore, "L'oublié de la science canadienne - George Lawson, 1827-1895», dans Pioneers of Canadian Science, op.cit. :54-80) ou de clarifier certains points de taxonomie et certains concepts (J. Rousseau et B. Boivin, «La contribution à la science de la Flore canadienne de l'abbé Provancher», Naturaliste canadien 95(1968):1499-1530; J.R. Barron, «Provancher's Collections of Insects...», Id. 102(4)(1975): 387591; W.O. Kupsch et W.A.S. Sarjeant (ed.), History of Concepts in Precambrian Geology, Toronto, 1979). Dans cette perspective, l'historien devient en quelque sorte la "conscience historique» de la science qui, non seulement met en ordre le passé, mais contribue, en outre, à maintenir la cohésion des institutions actuelles et à décourager la déviance individuelle en fournissant continuellement la preuve que, tôt ou tard, le mérite (ou le démérite...) de chacun sera reconnu.

En d'autres circonstances, l'histoire des sciences a été appelée, 
non plus uniquement à fournir des références et des modèles aux scientifiques du présent, mais à servir la cause de l'institutionnalisation et de la professionnalisation de la science dans la société canadienne. À titre d'exemple, on peut évoquer quelques-uns des multiples usages auxquels on a voulu faire servir la figure historique du Frère Marie-Victorin, é.c. Avant même sa disparition, le botaniste de l'Université de Montréal était devenu le symbole d'un réveil nationaliste et moderniste du Canada français où le progrès des sciences était défini comme la condition nécessaire et suffisante du progrès tout court (L.-P. Audet, Le Frère Marie-Victorin, éducateur. Ses idées pédagogiques, Montréal, 1942). Ce statut de symbole a été maintenu et même développé depuis car, s'emparant de son oeuvre scientifique et sociale, l'histoire traditionnelle s'est attachée à présenter Marie-Victorin, tour à tour ou simultanément, comme la preuve historique de la possibilité d'une réconciliation de la foi et de la science (Robert Rumilly, Le Frère MarieVictorin et son temps, Montréal, 1949), comme le champion d'un nationalisme «éclairé», c'est-à-dire "ouvert sur le monde et résolument tourné vers l'avenir» (Hermas Bastien, Marie-Victorin. Pour l'amour du Québec, Montréal, 1971) et comme le véritable fondateur de la science moderne au Québec (voir, entre autres, Pierre Dansereau, «La tradition botanique à Montréal», Culture 17(1956):379-387 et "L'héritage du Frère Marie-Victorin», Bulletin de la Société d'animation du Jardin botanique de Montréal 2(2)(1977):5-10). Par-delà les préférences de chaque biographe, privilégiant tel ou tel aspect particulier de son oeuvre et lui faisant jouer le rôle de pont, tantôt entre les sciences modernes et la religion, tantôt entre la rationalité scientifique et des idéologies politiques comme le nationalisme, la figure de Marie-Victorin a surtout servi de caution à la science elle-même, contribuant à la définition progressive des fonctions sociales, politiques et idéologiques du «savant» au Canada français, c'est-à-dire à la professionnalisation des activités de recherche et d'enseignement.

On peut citer facilement d'autres exemples où l'histoire des sciences au Canada a d'abord servi à la défense et à l'illustration de la science elle-même et, plus précisément, de ses institutions. Au National Research Council, on a entrepris depuis quelques années d'écrire l'histoire officielle de l'institution, en réaction aux vigoureuses attaques des universités et des milieux de la recherche industrielle, où on lui reprochait de monopoliser les ressources de l'État fédéral, et en réponse aux conclusions du Rapport Lamontagne (Rapport du Comité sénatorial de la politique scientifique, Ottawa, 1971 et s.) qui clouait le $N R C$ au pilori pour avoir historiquement privilégié la recherche fondamentale. Les travaux récents 
consacrés au $N R C$ par quelques auteurs qui ont voulu se porter à la défense de l'institution, tels Wilfrid Eggleston (National Research in Canada. The National Research Council, 1916-1966, Toronto, 1978), W.E.K. Middleton (Physics at the National Research Council of Canada, Waterloo, 1979), W.H. Cook (My Fifty Years with the NRC, 1924-1974, Ottawa, 1977) et N.T. Gridgeman (Biological Sciences at the NRC: The Early Years to 1952, Waterloo, 1979), doivent donc être envisagés dans ce contexte conflictuel. Ajoutons seulement que cette vaste entreprise de réfutation des critiques adressées au rôle historique du $N R C$ dans le développement des sciences au Canada, si elle a eu pour mérite de faire progresser les recherches sur une institution dont l'importance ne devrait pas être sous-estimée, n'a pas permis encore de bien cerner les principes de constitution et de fonctionnement des institutions scientifiques au sein de l'appareil d'État. C'est en tout cas l'avis de ceux qui ont été appelés à commenter l'histoire officielle du NRC (Paul Dufour, Isis 71(1980):658-659 et Donald Phillipson, HSTC Bulletin 13(1979):7-8). Aussi doit-on souhaiter que les recherches se poursuivent, moins liées à la conjoncture politique et à la réforme de l'appareil de l'État fédéral, et que soit mieux compris comment il se fait que l'État fédéral en soit venu à occuper une place prépondérante dans le financement, l'orientation et l'exécution de la science canadienne.

Dernier exemple où l'histoire traditionnelle des sciences et des techniques a puisé sa raison d'être dans la défense des institutions scientifiques et du «métier» de savant, l'ouvrage désormais classique de J.J. Brown sur l'histoire des techniques au Canada (Ideas in Exile. A History of Canadian Invention, Toronto, 1967) a servi d'inspiration non seulement aux avocats et aux théoriciens d'un «nationalisme économique» canadien qui s'appuyerait sur l'autonomie technologique nationale, mais, parallèlement, à ceux qui revendiquent pour la recherche scientifique et technique au Canada des moyens accrus. S'il est vrai, comme le prétend Brown, que les Canadiens ont maintes fois fait la preuve de leur génie inventif, mais n'ont pas su profiter de leurs propres inventions, au grand avantage des Américains, il incombe à l'État, dûment appuyé par l'opinion publique et l'initiative privée, de réunir les conditions qui permettraient aux inventeurs et aux entrepreneurs canadiens de se rencontrer enfin. Il revient à l'État également de mettre à la disposition des uns et des autres les moyens - capital de risque, subventions à la recherche, législation bienveillante à l'égard de l'innovation "autochtone» - nécessaires au renversement de la tendance historique à la fuite des cerveaux et des inventions, etc., etc. Nous voyons ici l'histoire des techniques fournir un fondement, au moins idéologique, aux politiques technologi- 
ques et économiques de l'État et servir du même coup les intérêts de ceux qui, les premiers, bénéficieraient d'un renforcement du potentiel canadien de recherche et développement ( $R$ et $D)$, c'est-àdire les ingénieurs et les scientifiques, à quelque secteur d'activité qu'ils appartiennent.

Ces quelques exemples suffisent, à notre avis, à illustrer la thèse selon laquelle l'histoire des sciences et des techniques au Canada, en tant que genre historiographique, a traditionnellement subordonné ses fins propres à la défense et à l'illustration de celles de la profession et des institutions de la science moderne. En ce sens, l'histoire des sciences, telle qu'elle a été faite jusqu'à présent, peut être assimilée à la vulgarisation scientifique, genre journalistique dont le but est moins de transmettre des savoirs objectifs et utiles que de diffuser une certaine image de l'activité scientifique et du savant ou, pour tout dire, de soigner les «relations publiques» de la science. Cette interprétation de l'ensemble de la production historiographique consacrée au développement des «rôles» de savant et d'ingénieur, des institutions et des différentes branches de la science et du génie, en tant que courant de recherche et d'analyse né des contraintes que rencontraient les processus de professionnalisation et d'institutionnalisation de la pratique scientifique au Canada, explique à la fois comment il se fait que les auteurs de cette histoire se sont traditionnellement recrutés parmi les hommes de science et les ingénieurs canadiens et pourquoi ceux-ci ont trouvé au sein de la communauté scientifique et dans des milieux étroitement associés à celle-ci leur public le plus fidèle. Cette interprétation permet également d'entrevoir en fonction de quoi la tradition historiographique a trouvé ses questions et ses problèmes dominants et quels facteurs l'ont empêché de se rendre autonome, en tant que discipline intellectuelle, par rapport au cadre problématique et institutionnel où elle a été progressivement formée, c'est-à-dire les «marges» des disciplines et des institutions de la science. Écrite par des scientifiques et pour des scientifiques, sa problématique commandée par l'état d'avancement de la pratique sociale de la science, l'histoire des sciences et des techniques au Canada aura été bien plus une discipline auxiliaire de la science que de l'histoire... Aussi, ne faut-il pas s'étonner de sa «marginalité» à l'égard des grands courants historiographiques et des grands problèmes de l'histoire du Canada, ni de l'ignorance relative dont ont fait preuve à son endroit jusqu'ici historiens et sociologues.

\section{L'émergence de questions nouvelles et quelques travaux récents}

Personne ne se surprendra, et les historiens moins que tout autre, de ce que certains travaux récents consacrés à l'histoire des 
sciences et des techniques au Canada continuent de servir la professionnalisation des «métiers» scientifiques, la nationalisation des activités de recherche et développement ou d'autres fins sociales, politiques et même franchement corporatistes, fins étrangères, en tout cas, à l'idéal de la pure connaissance historique. Non seulement les historiens des sciences continuent-ils de préparer bibliographies et biographies et se penchent-ils toujours sur les mêmes objets d'analyse (i.e. institutions, professions, disciplines et découvertes), mais encore un bon nombre d'entre eux tentent de répondre aux objectifs traditionnels. Dans certains milieux de recherche, l'histoire des sciences continue de s'écrire en fonction des politiques scientifiques et technologiques qu'entendent implanter l'État fédéral ou les gouvernements provinciaux. L'exemple donné il y a dix ans par les auteurs du célèbre Rapport Lamontagne, dans lequel quelques chapitres savamment travaillés passaient en revue l'histoire des sciences au Canada (voir, en particulier, les Chap. 2 à 5 du Vol. I, 1971), a été suivi, notamment par le Gouvernement du Québec dans le Livre vert sur la science (Pour une politique de la recherche scientifique, Québec, 1978) où l'on ne craint pas d'évoquer les mânes du Frère Marie-Victorin pour insuffler quelqu'ardeur nationaliste aux scientifiques québécois. Cela est de bonne guerre, semble-t-il, puisque l'Etat fédéral a, au même moment, tenté de mobiliser les historiens des sciences afin de dégager les composantes scientifiques et techniques de ce qui serait une authentique et originale culture canadienne. Prenant la suite du Rapport Symons (Se connaître: Le Rapport de la Commission sur les études canadiennes, Ottawa, 1975) qui recommandait chaudement le développement de l'histoire des sciences, le Conseil des Sciences du Canada a mis en train une vaste étude portant sur l'enseignement des sciences et la culture scientifique des Canadiens, étude dont un des objectifs essentiels est de favoriser la connaissance de l' "héritage scientifique et technique» du Canada (J.E. Page, Un contexte canadien pour l'enseignement des sciences, Ottawa, 1979 et G.S. Aikenhead, L'enseignement des sciences dans une perspective sociale, Ottawa, 1980). Comme on peut s'en douter, ces ouvertures de l'État fédéral, attendues parmi les universitaires, ont été accueillies avec reconnaissance et empressement par certains historiens des sciences et des techniques (R.A. Jarrell, "Why Do Many Canadians Know Nothing of Canadian Science?», Science Forum 10(1977):23-26; V. Shore, "Canadians Are Illiterate About Their Scientific Heritage», University Affairs (janvier 1979):6-8; etc.). Ce n'est pas notre intention de discuter des raisons qui poussent l'Etat fédéral à s'intéresser à la «culture scientifique» et à l'histoire des sciences et des techniques au Canada, ni des motifs qui conduisent des historiens à prêter leurs 
services à la reconstitution ou à l'évocation d'une telle chose que l'«héritage scientifique» du Canada. Cependant, il reste difficile d'imaginer que cette alliance qui se dessine ne liera pas pour longtemps encore une bonne part de la recherche historique à des entreprises idéologiques qui ont assez peu à voir avec l'avancement de la connaissance pour elle-même, ni avec une meilleure compréhension du rôle historique des sciences dans la formation de la société canadienne.

Malgré la permanence de problématiques et de contraintes anciennes, de nouvelles questions ont émergé au cours des dernières années et des travaux récents indiquent les avenues par lesquelles la recherche pourrait progresser pour s'affranchir de la tradition. Soulignons immédiatement que, parmi ces questions nouvelles, les plus intéressantes sont celles qui permettent de détacher la recherche d'objectifs plus ou moins étroitement liés à la pratique scientifique pour l'aligner sur les grandes interrogations de l'histoire sociale et économique du Canada.

Les historiens du développement économique du Canada, et tout particulièrement ceux qui ont marché sur les traces d'Harold Innis, ont généralement reconnu l'importance des techniques. Cependant, celles-ci ont surtout été considérées comme l'ensemble des formes que peut prendre, à chaque époque, le rapport de l'homme à la nature, rapport par lequel l'homme s'efforce de soumettre les forces naturelles et d'exploiter les ressources de son environnement. Dans cette perspective, les progrès des techniques deviennent condition générale de possibilités nouvelles: formes d'organisation du travail, formation de nouveaux arrangements industriels et commerciaux, apparition de nouveaux produits et de nouveaux processus, etc. Bref, le progrès technique devient condition de possibilité de ces innovations qui seraient, selon certaines doctrines économiques, la substance même du progrès économique et social. On trouvera dans des «classiques» de l'histoire économique canadienne, tels certains textes d'Albert Faucher (Québec en Amérique au XIX siècle, Montréal, 1973) et le livre de H.V. Nelles (The Politics of Development of Forests, Mines and HydroElectric Power in Ontario, 1849-1941, Toronto, 1974), l'exemple de ce parti-pris historiographique qui consiste à faire du processus d'innovation technique le facteur déterminant de l'histoire tout en s'interdisant de s'interroger sur la nature réelle de ce même processus et sur ses bases sociales. Sans entrer en opposition tranchée avec ce courant traditionnel de l'histoire économique canadienne, un intérêt nouveau se manifeste pour les techniques, qui les considère non plus sous le seul angle d'un rapport entre l'homme et la nature, mais aussi sous celui du rapport de l'homme à l'homme; ce 
qui est en train d'apparaître ici, c'est une histoire sociale des techniques, c'est-à-dire une analyse des innovations techniques telles qu'elles sont apparues dans une configuration sociale, dans un système de rapports de classes. Une telle histoire sociale et critique des techniques, intégrée à l'histoire économique, a pour but de définir à la fois à quel problème technique et à quels déterminismes sociaux chaque nouvelle machine, ou, plus globalement, chaque nouvelle étape du progrès des techniques répondait. De facteur initial et presque exogène, le progrès technique devient résultat du processus social dans son ensemble. Ajoutons que cette problématique nouvelle paraît s'accompagner d'un déplacement de la recherche historique des techniques artisanales aux techniques du mode de production industriel. Cependant, il faut reconnaitre que les travaux permettant d'illustrer la modification et le déplacement de la problématique en histoire de l'économie canadienne sont encore peu nombreux. Toutefois, nous pouvons citer à cette fin quelques chapitres des ouvrages de Fernand Harvey (Révolution industrielle et travailleurs, Montréal, 1978) et de Tom Naylor (The History of Canadian Business, 1867-1914, Toronto, 1975). Quant à savoir si une histoire proprement sociale des techniques au Canada peut faire changer le cours de l'histoire économique, c'est une question qui reste ouverte...

Après l'histoire économique, c'est sans doute l'histoire des idées et des cultures au Canada qui voit le plus s'élargir et s'enrichir son champ grâce aux travaux actuels des historiens des sciences. Avec les humanistes et les littéraires, les scientifiques du XIX ${ }^{e}$ et du $\mathrm{XX}^{\mathrm{e}}$ siècle ont puisamment contribué à forger la «culture canadienne» et à soutenir ces grands mouvements d'idées qu'ont été, parmi d'autres, l'impérialisme et le nationalisme et qui forment les cadres idéologiques principaux de l'histoire du Canada. Dans l'avenir, la recherche historique devrait tendre à mettre en lumière comment ont pu s'engendrer ou s'épauler mutuellement le rêve d'une science "canadienne» et la vision politique d'une jeune nation appelée à conquérir par la connaissance autant que par l'action un continent vierge; comment, encore, on a pu présenter le développement des études scientifiques et des recherches comme une des conditions de l'indépendance du Canada à l'égard de l'Europe et des États-Unis. Déjà, certains travaux ont dégagé le rôle joué, au siècle dernier, par des membres éminents de la communauté scientifique, tels W. Dawson et T.S. Hunt, dans la réassertion par l'«élite» canadienne d'un attachement indéfectible à l'Empire de concert avec les premières manifestations de nationalisme (V. de Vecchi, Science and Government in NineteenthCentury Canada, thèse de Ph.D., Université de Toronto, 1978), et dans la formation progressive d'une «identité nationale» à partir 
d'un curieux brassage d'idées politiques et scientifiques (A.B. McKillop, A Disciplined Intelligence. Critical Inquiry and Canadian Thought in the Victorian Era, Montréal, 1979). On peut citer également les travaux récents de sociologues qui ont éclairé la manière dont le scientisme et le populisme se sont conjugués, de 1920 à 1960, pour renouveler le vieux fond nationaliste du Canada français et contribuer à préparer la modernisation des institutions de la société québécoise au cours de la Révolution tranquille ( $\mathrm{F}$. Descaries-Bélanger, M. Fournier et L. Maheu, «Le Frère MarieVictorin et les petites sciences», Recherches sociographiques 20 (1)(1979):7-39). D'autres recherches précisent les conditions de développement de certaines branches du savoir et leur impact sur l'histoire des idées au Canada (par exemple, Yvan Lamonde, $L a$ philosophie et son enseignement au Québec, 1665-1920, Montréal, 1980). D'autres encore visent à cerner les particularités idéologiques et culturelles de l'activité scientifique et technique au Canada (T. Levere, "What is Canadian about Science in Canadian History?» dans Science, Technology, and Canadian History, op.cit., :14-22; Bruce Sinclair, «Canadian Technology: British Tradition and American Influences», Technology and Culture 20 (1979):108123).

Les historiens des sciences et des techniques ont également contribué aux progrès récents de l'histoire de la société canadienne et de ses institutions. Notamment, les rapports entre l'évolution de la structure sociale et le développement des professions scientifiques et techniques depuis le XIX ${ }^{\mathrm{e}}$ siècle ont été l'objet de nombreuses recherches: on pourra consulter, en particulier, les travaux de T.O. Eisemon et Y.M. Rabkin consacrés aux ingénieurs ( «Science in a Bilingual Society. The Case of Two Engineering Schools in Quebec», Social Studies of Science 8 (2)(1978):245-256 et «Spécificités nationales de la science et de la technologie: une étude de deux universités montréalaises», Recherches sociographiques 20 (1)(1979):87-101), ceux de Jacques Bernier sur la professionnalisation de la médecine («François Blanchet et le mouvement réformiste en médecine au début du XIX ${ }^{\mathrm{e}}$ siècle», Revue d'histoire de l'Amérique française 34 (2)(1980):223-244 et «Les praticiens de la santé au Québec, 1871-1921. Quelques données statistiques», Recherches sociographiques 20 (1)(1979):41-58) et ceux de Marcel Fournier et de Louis Maheu sur les sciences au Québec depuis 1920 («Le champ scientifique québécois», Sociologie et Sociétés 7 (1)(1975):119-132 et "Nationalisme et nationalisation du champ scientifique québécois», Sociologie et Sociétés 7 (2)(1975):89-114). La biographie collective (ou «prosopographie») des hommes et des femmes de science est également appelée à servir d'ici peu l'étude de la constitution de la communauté scientifique au XIX et au $\mathrm{XX}^{\mathrm{e}}$ 
siècles: en effet, un projet d'envergure dans ce domaine nouveau de l'histoire sociale des sciences et des techniques a été entrepris conjointement par l'Université de Toronto et l'Université de Montréal (cf. Philip Enros, «A Biobibliography of OntarioQuebec Scientists, 1914-1940», HSTC Bulletin 10(1979):3). Dans un contexte plus large, le rôle des sciences et des scientifiques dans la société canadienne commence à être mieux connu grâce à quelques études qui rompent radicalement avec une tradition vouée jusqu'ici à la défense et à l'illustration des savants et des institutions canadiennes. Parmi ces études récentes d'histoire sociale des sciences, celles illustrant le mieux les tendances nouvelles sont celles de R.A. Jarrell («The Rise and Decline of Science at Quebec, 1824-1844», Histoire sociale 10(1977):77-91 et «Science Education at the University of New Brunswick in the Nineteenth-Century», Acadiensis (1973):55-79) et de Peter J. Bowler («The Early Development of Scientific Societies in Canada», dans A. Oleson et S.C. Brown (ed.), The Pursuit of Knowledge in the Early American Republic, Baltimore, 1976 :326-339). On peut citer également l'analyse que font R. Daley et P. Dufour de la fondation de la Société royale du Canada ("Creating a 'Northern Minerva': John William Dawson and the Royal Society of Canada», HSTC Bulletin 17(1981):3-14). Enfin, ceux qui étudient l'histoire politique du Canada et de ses provinces trouveront sans doute quelque profit dans les travaux consacrés aux rapports entre l'État et les institutions scientifiques canadiennes, tout spécialement, dans les ouvrages de V. de Vecchi (Science and Government..., op.cit.), de Bruce G. Doern (Science and Politics in Canada, Montréal, 1972) et de R. Duchesne (La science et le pouvoir au Québec, 1920-1965, Québec, 1978). Au sujet des rapports entre l'Etat et la science, la bibliographie de l'Institut canadien de l'information scientifique et technique (La politique des sciences, la recherche et le développement au Canada, op.cit.) livrera de nombreux titres supplémentaires.

Il faut signaler un autre domaine où les recherches se multiplient depuis quelque temps; celui des études comparées du développement des sciences et des techniques dans le cadre de l'expansion européenne depuis la Renaissance. Soulignons que les études proprement canadiennes rejoignent ici une problématique universelle qui met en question les facteurs et les conditions de la diffusion des sciences et des techniques modernes, depuis les pays où elles ont pris origine à travers les continents et les cultures. Parmi les travaux qui s'attaquent, à partir du cas du Canada, à ce problème de la professionnalisation et de l'institutionnalisation des sciences dans une société coloniale, où les contraintes de dépendance politique, économique et culturelle imposent des conditions 
particulières au progrès des sciences, mentionnons, en premier lieu, le travail déjà ancien de Donald Fleming, qui ouvrait la voie aux études comparées ( Science in Australia, Canada, and the United States: Some Comparative Remarks», Actes du $10^{e}$ Congrès international d'histoire des sciences, Paris, 1964 :179-196). Cet exemple a été suivi, notamment, par Lewis Pyenson («The Incomplete Transmission of a European Image: Physics at Greater Buenos Aires and Montreal, 1890-1920», Proc. of the American Phil. Society 122 (2)(1978):92-114 et par R.A. Jarrell («Two Scientific Revolutions That Failed: The Truncation of Science and Colonialism in Ireland and French Canada», HSTC Bulletin 18 (1981):150-165). Citons également, parmi ceux qui se sont intéressés à la question du développement des sciences au Canada en tant que société périphérique ou dépendante, V. de Vecchi (op.cit.), L. Pyenson et Susan Sheets-Pyenson («The Formative Years of Canadian Science: John William Dawson and his Circle, 18651886», communication présentée à la Société canadienne d'histoire et de philosophie des sciences à Saskatoon, 1979, poly.), Yves Gingras («La physique à McGill entre 1920 et 1940: la réception de la mécanique quantique par une communauté périphérique», HSTC Bulletin 17 (1981):15-39, et R. Duchesne («Science et société coloniale: les naturalistes du Canada français et leurs correspondants scientifiques, 1860-1900», HSTC Bulletin 18 (1981):99-139). Ces problèmes de la transmission et de l'adaptation des pratiques scientifiques et techniques dans des sociétés nouvelles sont trop importants, à la fois pour la compréhension de la situation actuelle des sciences dans les pays du Tiers monde et pour l'analyse du développement historique des sciences dans des pays comme les États-Unis ou l'Australie, qui ont, comme le Canada, un passé colonial, pour qu'ils ne continuent pas d'être l'objet de nombreuses études dans l'avenir.

En dernier lieu, on peut ajouter que cette émergence de nouvelles questions et de nouvelles recherches, dont nous venons de souligner les principaux aspects, s'accompagne d'un regain d'intérêt pour les sources d'archives et que les historiens des sciences et des techniques ont généralement trouvé chez les archivistes une conscience éveillée de la valeur des documents témoignant du passé scientifique et technique du Canada. Déjà à Kingston, en 1978, historiens et archivistes ont eu l'occasion de discuter de questions telles que la sélection, l'acquisition et la conservation de fonds d'archives concernant soit des individus, soit des sociétés ou des institutions de recherche et d'enseignement (Sandra Guillaume, «Problems Faced by Archivists Dealing with Historical Resources of Science and Technology in Canada», dans Science, Technology, and Canadian History, op.cit. :177-183). Aux Archives 
publiques du Canada ainsi que dans certaines institutions comme le National Research Council, la présence d'archivistes spécialement chargés de veiller sur les collections scientifiques et techniques témoigne de l'importance que l'on accorde désormais à cet héritage historique. À l'Université de Montréal, à l'Université McGill, à l'Université de Toronto, etc., bref, dans ces institutions qui ont été les principaux piliers du développement des sciences au Canada, les fonds et collections d'archives, déjà considérables parfois, continuent de s'accroître. Enfin, il faut citer le travail exemplaire accompli par Gilles Janson, de l'Université du Québec à Montréal, qui non seulement a entrepris de rechercher et de décrire les archives médicales de la région de Montréal (G. Janson et $M$. Lalonde, "Guide des sources d'archives concernant la médecine sur l'île de Montréal, des débuts jusqu'à 1900», à paraître dans Archivaria), mais qui a pu, en outre, réunir dans son institution les archives des plus importantes sociétés savantes du Canada français; l'ACFAS, la Société de biologie de Montréal, les Cercles des jeunes naturalistes, etc. On ne saurait d'aucune manière sous-estimer l'importance du rôle que sont appelés à jouer archivistes et dépôts d'archives dans l'élargissement et le renouvellement des problématiques de l'histoire des sciences et des techniques au Canada.

\section{En conclusion}

Où en est l'histoire des sciences et des techniques au Canada? Pour répondre de manière succincte à cette question, disons qu'elle se trouve à ce point crucial de son évolution où ses interrogations et ses fins traditionnelles, liées aux pratiques scientifiques, se trouvent progressivement reléguées au second rang par l'émergence de problèmes nouveaux, plus directement inspirés par l'histoire économique et sociale du Canada. Les historiens de la première heure, les Tory, les Rousseau, les Lortie, les Eggleston, nous ont donné des travaux d'une incontestable valeur et certaines de leurs analyses continueront pendant longtemps encore de faire l'unanimité parmi les historiens. On peut prévoir également qu'une part considérable de la recherche historique sur les savants et les institutions scientifiques du Canada continuera de trouver sa raison d'être dans la défense et l'illustration de la science. Cependant, il apparaît clairement que le progrès de l'histoire des sciences et des techniques au Canada, en tant que discipline historique, dépendra essentiellement du travail de chercheurs rompus aux méthodes nouvelles de la recherche historique et du ré-alignement des problématiques sur celles de l'histoire du Canada. C'est à cette double condition que l'histoire des sciences et des techniques pourra apporter sa contribution à l'interprétation de l'expérience canadienne. 